\title{
RECENT DEVELOPMENTS ON BEAM OBSERVATIONS AT SRRC
}

\author{
Ian C. Hsu, G. H. Luo, and K. T. Hsu \\ Department of Nuclear Science, National Tsing-Hua University and \\ Synchrotron Radiation Research Center, \\ Hsinchu, 300 Taiwan
}

\begin{abstract}
In this paper, we will present some recent developed methods of beam observations and their experimental results. These methods including: beam energy measurement by laser Compton scattering, investigating electron beam orbit sensitivities on the flux of the photon beam line, beam lifetime investigation in storage rings.
\end{abstract}

\section{INTRODUCTION}

We have been trying to utilize the known principles of Beam physics and the existing Photonics knowledge and devices to investigate or to perform new methods of beam observations. In this paper, we will present some recent developed methods of beam observation and their experimental results. These methods including: beam energy measurement by laser Compton scattering, investigating electron beam orbit sensitivities on the flux of the photon beam line, beam lifetime investigation in storage rings. Only brief description and results are presented in this review. References are provided for more detail information for each individual study. All of the experiments are performed on the electron beam in the storage ring of Taiwan Light Source(TLS) of Synchrotron Radiation Research Center(SRRC), Taiwan.

\section{BEAM ENERGY MEASUREMENT BY LASER COMPTON SCATTERING[1],[2]}

The method of Compton scattering to measure the electron beam energy in the storage ring or to produce quasi-monochromatic $\gamma$-rays is characterized by excellent signal-to-noise ratio. To acquire a high $\gamma$-ray flux, a pulsed $\mathrm{CO}_{2}$ laser with up to $2.67 \mathrm{MW}$ peak power is employed. Owing to the fact that the background radiation from Bremsstrahlung is extremely high ( about 1200 counts/sec at $20 \mathrm{~mA}$ electron beam current ), how to effectively subtract the background radiation is a relevant concern. In this study, we developed the method of synchronous measurement to resolve the above problem. The synchronous measurement used a gate to periodically allow the signals to pass from the detector to the counting system. Since the scattered photons were produced after the laser pulse reached the interaction region, the laser could provide a trigger signal for the gate to open. The method proposed herein increases the signal to noise ratio from 1.2 to 42.5 . Figure 1 presents the entire system's schematic diagram. According to this figure, the laser photons pass through the optical system into the storage ring's straight section. After being scattered by relativistic electrons, the $\gamma$-rays passes through the lead collimator and is then detected by the HPGe detector. The signal processing instruments, then, acquire the backscattered $\gamma$-rays' spectrum.

Considering that the highest energy of the backscattered photons was around $3000 \mathrm{keV}$, we chose ${ }^{24} \mathrm{Na}$ the standard source in energy calibration of the HPGe detector since the two characteristic energies of ${ }^{24} \mathrm{Na}$ were $1368.4 \mathrm{keV}$ and $2753.6 \mathrm{keV}$. Those energies contributed to a sum-peak energy of $4122 \mathrm{keV}$ that could be applied to the interpolation method in energy calibration.

Figure 2 presents the spectrum of the Compton scattering with a collimator having an inner diameter of $3 \mathrm{~mm}$ that corresponded to a half opening angle of $0.2241 \mathrm{mrad}$. The background radiation's counting rate without the laser Compton scattering effect was around 0.82 counts/sec with gating. After the laser collided with the electron beams, the counting rate raised to 34.83 counts/sec. The $S / N$ ratio was approximately 42.5 .

The highest back-scattered $\gamma$-ray energy could be estimated from the sharp edge of the spectrum as shown in Fig. 2. For our latest experiment, it was $3054 \mathrm{keV} \pm 2.6 \mathrm{keV}$. According to the results, we can infer that the electron beam energy was $1.3058 \pm 0.0017 \mathrm{GeV}$. The relative energy measurement uncertainty of this experiment is $0.13 \%$.

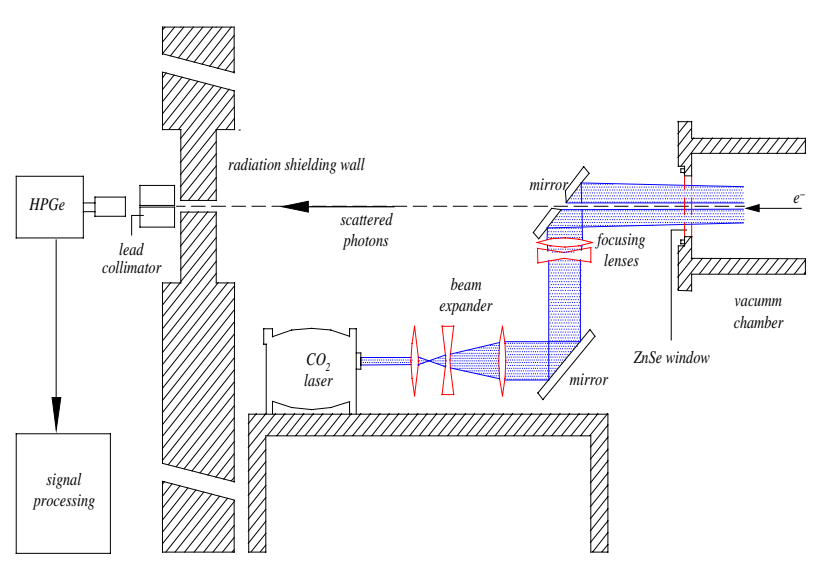

FIG. 1 Schematic diagram of the overall system: part of the vacuum chamber of the storage ring, optical system, detecting system, and signal processing system. 


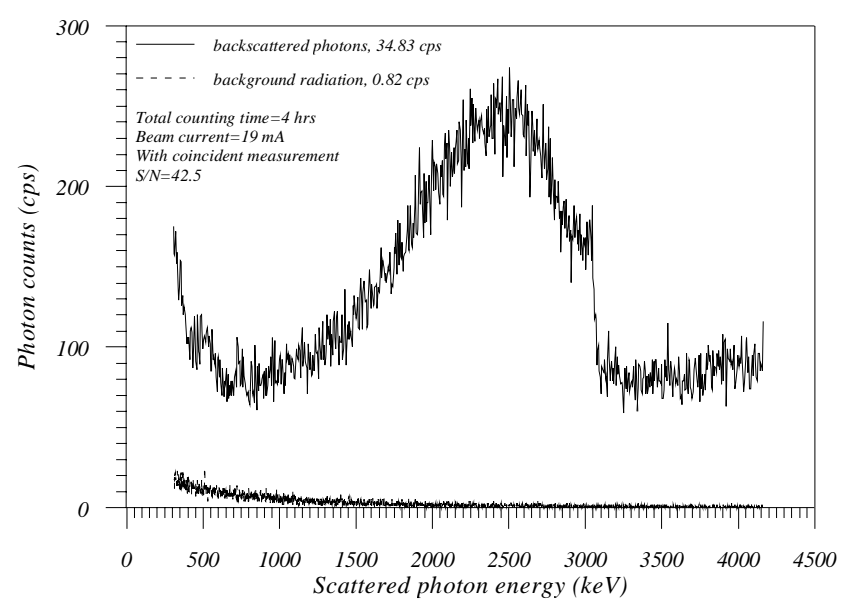

FIG. $2 \gamma$-ray spectrum of Compton scattering with collimator of $3 \mathrm{~mm}$ diameter under synchronous measurement. (electron beam current $=19 \mathrm{~mA}$, counting time $=4 h r s$, and $S / N$ ratio $=42.5$.)

\section{INVESTIGATING ELECTRON BEAM ORBIT SENSITIVITIES ON THE FLUX OF THE PHOTON BEAM LINE[3]}

This study examines the beamline flux sensitivity due to an electron beam's positional and angular changes at the source point of the beamline. Beam experimental and numerical studies have been undertaken. It was performed by varying the size of either the electron beam's orbit local position bump or that of the local angular bump. Changes in the beamline flux are measured at the entrance slit downstream. Those two types of local bumps are created by four correction magnets. The strength of four correction magnets must adhere to a certain ratio to control the amplitude and slope of the electron beam's orbit at a given position in a ring. The experiments in this study are conducted on the 6m-HSGM (6 meter High energy Spherical Grating Monochromator)[4] beamline at TLS.

Results obtained from the beamline studied herein indicate that $10 \mu \mathrm{m}$ vertical beam position displacement causes a relative photon flux change of $0.9 \pm 0.3 \%$, as measured at the entrance slit downstream. This observation corresponds to the numerical results. In addition, a vertical beam angular change of $10 \mu \mathrm{rad}$ causes a relative photon flux change of $1.2 \pm 0.4 \%$. The above two values depend on the electron beam size, slit size as well as the beamline's optics. Figure 3 presents the measurement results for which only the position bump was varied. Horizontal axis denotes the beam position at the source point. The left vertical axis represents the relative photon flux fluctuation $\left(\Delta \mathrm{I}_{\mathrm{o}} / \mathrm{I}_{\mathrm{o}}\right)$ per unit beam position displacement at the source point. While varying the beam positions, the beam angle should remain unchanged. Due to the position bump's imperfection, the beam angle at the source point slightly changes. This figure also plots the beam angle's value at each step on the right vertical axis, i.e. the $\mathrm{X}-\mathrm{X}^{\prime}$ correlation.

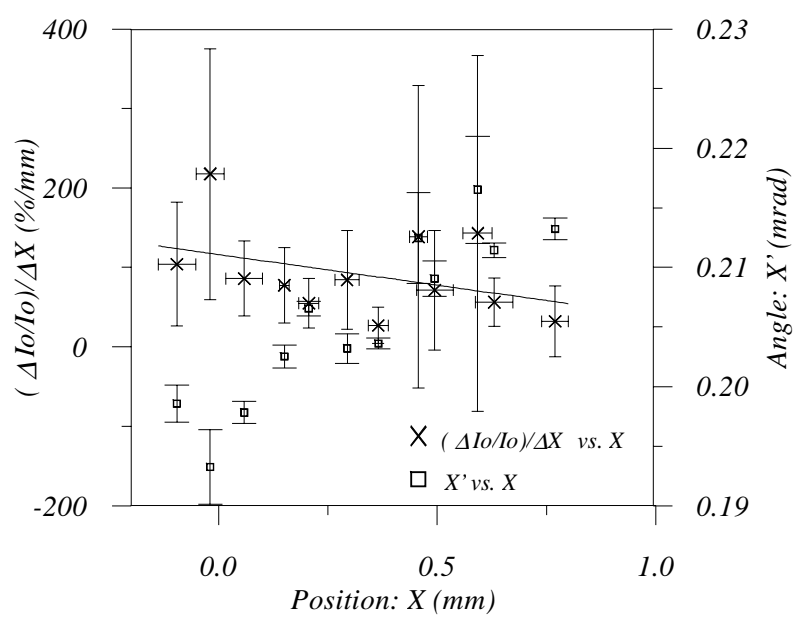

Fig. 3 The measurement results for which only the position bump was varied.

\section{BEAM LIFETIME INVESTIGATION IN STORAGE RINGS[5]}

As is generally known, the two unequal bunches method[6] can separate the beam lifetime contributions from these two effects. The beam current vs. time for each individual bunch was measured by the voltage signal of a broad band pickup. The lifetime of each individual bunch was then calculated. The calibration of the broad band pickup signal was done by a DC current transformer (DCCT) when a single bunch beam was stored. Figure 4 summarizes these results. The horizontal axis is the difference between the two bunch currents in mA. The vertical axis is the difference of the inverse of the bunch total lifetime. From the slope of the fitted line, the proportionality constant $\mathrm{A}$ can be obtained and the Touschek lifetime for a given bunch current can be calculated. Under the experimental conditions of the nominal transverse beam sizes $\left(\sigma_{\mathrm{x}}=180 \pm 9 \mu \mathrm{m}, \sigma_{\mathrm{y}}=\right.$ $70 \pm 5 \mu \mathrm{m}$ ), a total RF voltage of $700 \mathrm{kV}$ and a bunch length of $100 \mathrm{ps}$, the results derived from Fig. 4 are:

Touschek lifetime $(\min )=(366 \pm 52) /($ single bunch current in $\mathrm{mA})$

In the following calculations, the above results are used to estimate the multibunch beam lifetimes. For a total beam current of $195 \mathrm{~mA}$ (filling 140 bunches), the single bunch current is $1.39 \mathrm{~mA}$. The Touschek lifetime is $263 \pm 38$ $\mathrm{min}$. The measured total beam lifetime at $195 \mathrm{~mA}$ with a multibunch mode by DCCT is $238 \pm 1$ min. Subtracting $1 /(263 \mathrm{~min})$ from $1 /(238 \mathrm{~min})$ yields the gas scattering lifetime as $2500 \pm 1361 \mathrm{~min}$. The reason of the large uncertainty was discussed in Ref[5]. 


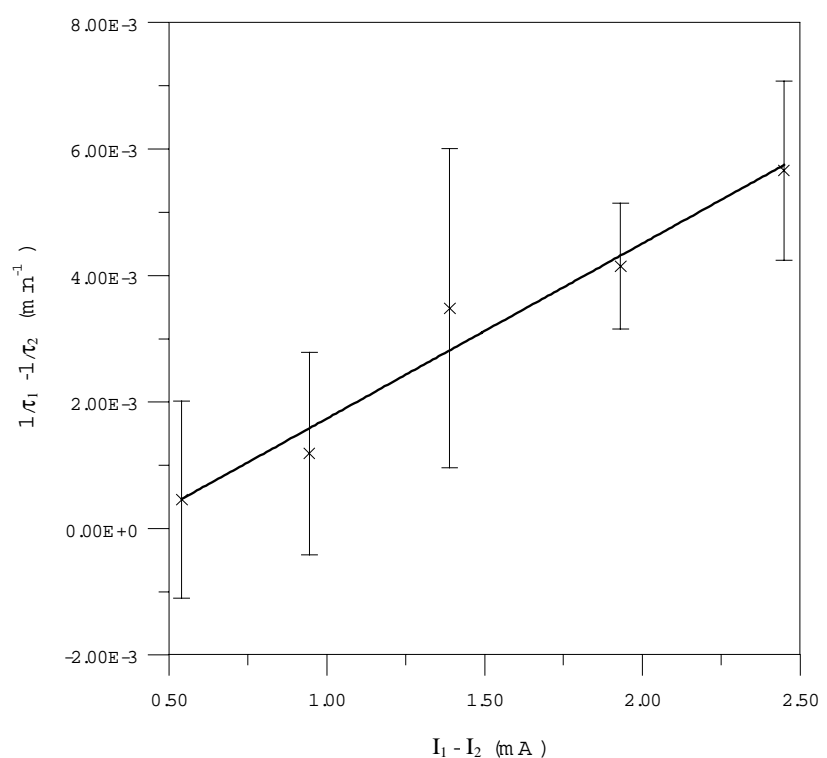

Fig. 4 The Touschek lifetime measurement by the two unequal bunch beam method. The horizontal axis is the difference between the two bunch currents in units of $\mathrm{mA}$.

The vertical axis is the difference of the inverse of the bunch total lifetime.

In the multibunch method, using the same total RF voltage, we first measured the total beam lifetime at the multibunch mode with the nominal transverse beam sizes $\left(\sigma_{\mathrm{x}}=180 \pm 9 \mu \mathrm{m}, \sigma_{\mathrm{y}}=70 \pm 5 \mu \mathrm{m}\right)$. The beam current was $195 \mathrm{~mA}$ (filling 140 bunches) with a total beam lifetime $\tau_{\mathrm{a}}$ of $238 \pm 1 \mathrm{~min}$. Next, the beam was driven into the difference resonance and the transverse beam area $\left(\sigma_{\mathrm{x}}=\right.$ $\left.357 \pm 14 \mu \mathrm{m}, \sigma_{\mathrm{y}}=353 \pm 16 \mu \mathrm{m}\right)$ was increased by a factor of 10. The beam current was $194.3 \mathrm{~mA}$ with a total beam lifetime $\tau_{10 \mathrm{a}}$ of $960 \pm 1 \mathrm{~min}$. If the difference between the beam current of $195 \mathrm{~mA}$ and $194.3 \mathrm{~mA}$ can be disregarded, the following two equations can be solved easily, yielding a Touschek lifetime $\tau_{\mathrm{T}}$ of $285 \pm 14 \mathrm{~min}$ and a gas scattering lifetime $\tau_{\text {gas }}$ of $1443 \pm 280$ min which includes the ion effects, if they exist.

$$
\begin{aligned}
& 1 / \tau_{\mathrm{a}}=1 / 238=1 / \tau_{\mathrm{T}}+1 / \tau_{\mathrm{gas}} \\
& 1 / \tau_{10 \mathrm{a}}=1 / 960=1 /\left(10 \tau_{\mathrm{T}}\right)+1 / \tau_{\mathrm{gas}} \\
& \text { (2) }
\end{aligned}
$$

The results obtained above are valid for estimating the beam lifetimes for the multibunch operation mode because all the measurements are taken in the multibunch mode.

According to the measurement results, the Touschek lifetime is longer when measured by the multibunch method ( $285 \pm 14 \mathrm{~min})$ than when measured by the two unequal bunches method $(263 \pm 38 \mathrm{~min})$. The reason for this discrepancy is that in the two unequal bunches method, the Touschek lifetime was measured at the few bunches mode and in that mode, the bunch lengthening effects, possibly caused by the couple bunch effects may not be as prevalent as those in the multibunch mode. For the estimation of the gas scattering lifetime of the operation mode i.e., the multibunch mode, in which the total lifetime was measured, we used the Touschek lifetime measured at the few bunches mode. This misuse caused the Touschek effect in the multibunch mode to be overestimated and consequently, underestimated the gas scattering effects in the multibunch mode. The Touschek lifetime is longer by $8 \%$ \{i.e., $(285-263) /[(285+263) / 2]$ \} when measured by the multibunch method than when measured by the two unequal bunches method. The theoretical value of the Touschek lifetime at the corresponding parameters is $302 \mathrm{~min}$. There is a $54 \%$ \{i.e., $(2500-1443) /[(2500+1443) / 2]\}$ discrepancy of the gas scattering lifetime measured by the two methods. Besides the consequence of the overestimation of the Touschek effect as previously mentioned, a significant reason for this discrepancy is a possibility of the discounting of the effects of any trapped ions in the multibunch operation mode, when the lifetimes were estimated by the results of the two unequal bunches method.

\section{ACKNOWLEDGMENTS}

We would like to thank Prof. Y. C. Liu and Dr. G. L. Chen and the Operation group, the Vacuum group of SRRC for their help while we are doing the experiment. The support of SRRC and NSC, Taiwan was acknowledged.

\section{REFERENCE}

[1] I. Hsu, C.-C. Chu, and C. I. Yu, Phys. Rev., E 54(5), 5657 (1996).

[2] I. Hsu, C.-C. Chu, S.-S. Chen C.-L. Cho, and H.-C. Chen, Nucl. Instrum. and Meth. A 384, 307(1997).

[3] I. Hsu, Phys. Rev., E 55(5), 6011 (1997).

[4] S. C. Chung et al., Rev. Sci. Instrum. 66(2), 1655 (1995).

[5] I. Hsu, Jpn. J. Appl. Phys. 36, 11 (1997).

[6] J. C. Besson, P. Certain, A. Dael, P. Juan, A. Labeque, M. P. Level, P. C. Marin, J. Michaut, C. MonetDescombey, P. Nghiem, M. Sommer, R. Souchet and H. Zyngier: ORSAY Rep. RT-88-01, France, 1980. 